\title{
Prediction of plant pre-microRNAs and their microRNAs in genome-scale sequences using structure-sequence features and support vector machine
}

\author{
Jun Meng ${ }^{1}$, Dong Liu', Chao Sun ${ }^{1}$ and Yushi Luan ${ }^{2 *}$
}

\begin{abstract}
Background: MicroRNAs (miRNAs) are a family of non-coding RNAs approximately 21 nucleotides in length that play pivotal roles at the post-transcriptional level in animals, plants and viruses. These molecules silence their target genes by degrading transcription or suppressing translation. Studies have shown that miRNAs are involved in biological responses to a variety of biotic and abiotic stresses. Identification of these molecules and their targets can aid the understanding of regulatory processes. Recently, prediction methods based on machine learning have been widely used for miRNA prediction. However, most of these methods were designed for mammalian miRNA prediction, and few are available for predicting miRNAs in the pre-miRNAs of specific plant species. Although the complete Solanum lycopersicum genome has been published, only 77 Solanum lycopersicum miRNAs have been identified, far less than the estimated number. Therefore, it is essential to develop a prediction method based on machine learning to identify new plant miRNAs.

Results: A novel classification model based on a support vector machine (SVM) was trained to identify real and pseudo plant pre-miRNAs together with their miRNAs. An initial set of 152 novel features related to sequential structures was used to train the model. By applying feature selection, we obtained the best subset of 47 features for use with the Back Support Vector Machine-Recursive Feature Elimination (B-SVM-RFE) method for the classification of plant pre-miRNAs. Using this method, 63 features were obtained for plant miRNA classification. We then developed an integrated classification model, miPlantPreMat, which comprises MiPlantPre and MiPlantMat, to identify plant pre-miRNAs and their miRNAs. This model achieved approximately $90 \%$ accuracy using plant datasets from nine plant species, including Arabidopsis thaliana, Glycine max, Oryza sativa, Physcomitrella patens, Medicago truncatula, Sorghum bicolor, Arabidopsis lyrata, Zea mays and Solanum lycopersicum. Using miPlantPreMat, 522 Solanum lycopersicum miRNAs were identified in the Solanum lycopersicum genome sequence.
\end{abstract}

Conclusions: We developed an integrated classification model, miPlantPreMat, based on structure-sequence features and SVM. MiPlantPreMat was used to identify both plant pre-miRNAs and the corresponding mature miRNAs. An improved feature selection method was proposed, resulting in high classification accuracy, sensitivity and specificity.

Keywords: MiRNA, Pre-miRNA, Prediction, SVM, Feature selection

\footnotetext{
* Correspondence: luanyush@dlut.edu.cn

${ }^{2}$ School of Life Science and Biotechnology, Dalian University of Technology,

Dalian, Liaoning 116023, China

Full list of author information is available at the end of the article
}

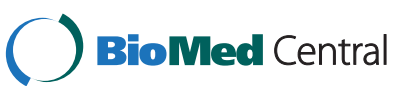

(c) 2014 Meng et al.; licensee BioMed Central. This is an Open Access article distributed under the terms of the Creative Commons Attribution License (http://creativecommons.org/licenses/by/4.0), which permits unrestricted use, distribution, and reproduction in any medium, provided the original work is properly credited. The Creative Commons Public Domain Dedication waiver (http://creativecommons.org/publicdomain/zero/1.0/) applies to the data made available in this article, unless otherwise stated. 


\section{Background}

MicroRNAs (miRNAs) are a family of non-coding RNAs approximately 21 nucleotides (nt) in length that play important roles at the post-transcriptional level in animals, plants and viruses [1]. These molecules are first cut from a stem-loop structure by RNaseDicer III. Environmental stress can induce or repress the expression of some miRNAs, thereby regulating the expression of downstream genes that respond to environmental stresses. The initial products of miRNA gene transcription are pre-miRNAs. Next, enzymes release pre-miRNAs with hairpin structures of 53-938 nt [2] by cutting and splicing. Finally, mature miRNAs are released from pre-miRNAs with hairpin structures by Dicer-like enzyme.

Mature miRNAs combine with RISC protein complexes to target specific mRNAs [3] and induce gene silencing by mRNA degradation or transcriptional inhibition. Plant miRNAs target multiple sites [4] to regulate various aspects of plant growth and development, including cell growth, cell differentiation, root, stem, leaf and other morphologies; these miRNAs also function in plant adaptation to different biotic and abiotic conditions $[5,6]$.

The methods used to predict the role of miRNAs can be divided into two categories: experimental verification and bioinformatic prediction. Although experimental verification, which is based on direct cloning experiments, can identify many miRNAs with high expression levels, few miRNAs with low or specific expression can be identified. Moreover, this method is expensive and results in a high number of false positive results. Bioinformatic prediction can compensate for these deficiencies. Based on recent studies, bioinformatic methods for identifying miRNAs can be divided into three categories: alignment analysis, machine learning and high-throughput sequencing $[7,8]$. Studies have shown that miRNAs are conserved among species. Pre-miRNAs containing mature miRNAs can be folded to form hairpin structures that have low minimum free energy (MFE) values [9]. Alignment analysis is based on these properties. MiRscan [10], miRFinder [11] and miREval [12], which are based on alignment homology analysis, have been successfully applied. Due to a lack of miRNA structural information, most of these methods yield high false positive rates. Based on prior knowledge, appropriate data are selected, appropriate features are chosen, and a high-performance data-mining algorithm is used to construct a classification model. Triplet-SVM [13], bayesmiRNAfind [14] and MiPred [15] are successful models that are based on the machine learning method. However, few of these models can be used for plant pre-miRNA prediction because the hairpin structure of plant pre-miRNAs is much more complex than that of animal pre-miRNAs. Moreover, these models cannot be used to predict mature miRNAs in specific species [16]. High-throughput sequencing identifies not only pre-miRNAs but also mature miRNAs [17]. An integrated model to identify plant miRNA-target interactions has been proposed [18]. However, due to the existence of genome-wide sequencing errors, mistakes may occur when comparing with short sequences. Furthermore, some parameters are set based on experience and lack a strong theoretical basis. There is no consensus regarding miRNA prediction.

In this study, we focus on building a model that can be used in the classification of real/pseudo plant premiRNAs together with their mature miRNAs via the machine learning method. An initial set of 152 novel features related to sequential structure was used in the model. By applying feature selection, the subset of 47 features yielding optimal results was obtained using Back Support Vector Machine-Recursive Feature Elimination (B-SVM-RFE) in real/pseudo plant pre-miRNA classification. In the same way, the subset of 63 features yielding optimal plant miRNA classification was obtained. An integrated classification model, miPlantPreMat, was trained to identify real/pseudo plant pre-miRNAs and the corresponding miRNAs. MiPlantPreMat achieved high accuracy on plant datasets from nine plant species, including Arabidopsis thaliana, Glycine max, Oryza sativa, Physcomitrella patens, Medicago truncatula, Sorghum bicolor, Arabidopsis lyrata, Zea mays and Solanum lycopersicum. For example, 522 Solanum lycopersicum miRNAs were obtained from the Solanum lycopersicum genome sequence. The superior performance of the proposed classifier can be attributed to the extraction of plant pseudo pre-miRNAs, selection of the training dataset and careful feature selection. The website dedicated to miPlantPreMat includes the training and testing datasets, training models (MiPlantPre and MiPlantMat) and miPlantPreMat source codes used, all of which are freely available (https://github.com/kobe-liudong/miPlantPreMat). We provide a detailed description of the sources used for the datasets in the readme.txt in the 'data' folder.

\section{Methods}

\section{Dataset preparation for the training and testing of the} SVM model

An effective classifier of plant pre-miRNAs and miRNAs should distinguish real pre-miRNAs and miRNAs from pseudo pre-miRNAs and miRNAs. The positive dataset comprised known plant pre-miRNAs and miRNAs, whereas the negative dataset comprised pseudo Solanum lycopersicum, pseudo Glycine max and pseudo Arabidopsis lyrata hairpins.

All 6,378 plant miRNAs and 5,166 plant pre-miRNAs that were experimentally verified in miRBase release 19.0 were screened for inclusion in the positive data set. Redundant sequences were excluded, and the remaining nonredundant sequences were folded into hairpin secondary 
structures. Of these pre-miRNAs, 3,126 non-redundant pre-miRNAs having single stem-loops were treated as real samples for miPlantPre. Plant pre-miRNAs range from $53 \mathrm{nt}$ to $938 \mathrm{nt}$ in length and have more complex secondary structures than mammalian pre-miRNAs. It is difficult to locate the position of miRNAs and miRNAs* for plant pre-miRNAs. In this study, pre-miRNAs were intercepted such that mature miRNAs in pre-miRNAs are at the 3'- or 5 '-end of the selected sequence. We treated these premiRNAs as real samples within miPlantMat. Furthermore, pre-miRNAs intercepted at other positions were treated as pseudo samples within miPlantMat. After interception, the pre-miRNAs exhibited a narrower length range, and structures in each region of the pre-miRNAs were more unified. The 152 features applied in miPlantPre were also used because the stem-loop structure was maintained. In this process, the proposed features termed MFEI7, MFEI8, MFEI9, Mis_num_begin, Mis_num_end and "G(((_begin_S", "A.(._end_S" were useful because they helped to identify real pre-miRNAs that were intercepted at different positions. For consistency, all pre-miRNA secondary structures were recalculated using RNAfold in the Vienna package [19]. Figure 1 illustrates the interception procedure using the stem-loop of Solanum lycopersicum miR$166 \mathrm{~b}$ as an example. The length was shortened from
$201 \mathrm{nt}$ to $138 \mathrm{nt}$ by removing the bases before the miRNA* and after the miRNA.

Almost all reported miRNAs are located in untranslated regions or intergenic regions, although some can be found in protein-coding sequences (CDSs). Some sequence segments have stem-loop structures that are similar to those of real pre-miRNAs but have not been reported as premiRNAs. Because the model was trained to distinguish analogous real/pseudo pre-miRNAs, the sequences in the negative dataset should regard structures with stem-loop structures as genuine pre-miRNAs; otherwise, the classification will not yield significant results. CDSs of Solanum lycopersicum, Glycine max and Arabidopsis lyrata RefSeq genes with no known alternative splice events were collected. Most known plant pre-miRNAs are $120 \mathrm{nt}$ in length. Thus, a sliding window of widths ranging randomly from 60 to $150 \mathrm{nt}$ was used to scan the CDSs to produce sequence segments. The sequence segments should fold into single stem-loop structures and satisfy five criteria based on the number of base pairs in hairpins, $\% \mathrm{G}+\mathrm{C}$, MFEI, the complementary base pairing of mature miRNAs and the stability of the precursor in relation to the MFE rate. The criteria were determined by observing real intercepted plant pre-miRNAs. The criteria for selecting pseudo miRNAs were as follows: a minimum of 19

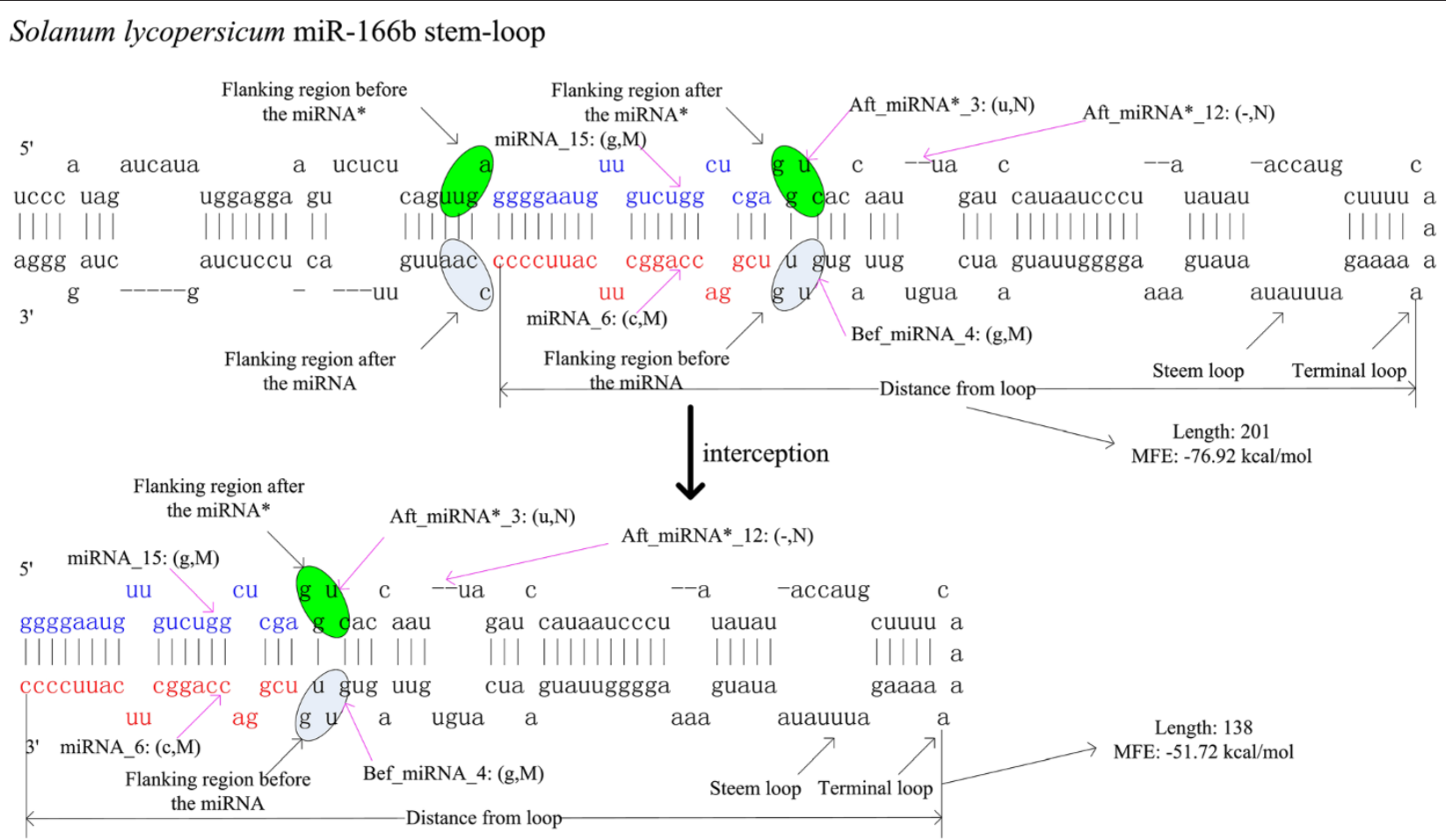

Figure 1 Original pre-miRNA and intercepted pre-miRNA of Solanum lycopersicum miR-166b. Mature miRNA is at $3^{\prime}-e n d$ and miRNA* $^{*}$ is at $5^{\prime}$-end of the selected sequence. Each base has two states, match or mismatch. Each precursor contains one loop at least. The original pre-miRNA has 201 bases with the MFE $-76.92 \mathrm{kcal} / \mathrm{mol}$ and the intercepted pre-miRNA has 138 bases with the MFE $-51.72 \mathrm{kcal} / \mathrm{mol}$. 
base pairings in the hairpin structure, \%G $+\mathrm{C}>0.242$ and $<0.825$, MFEI $>0.522$ and $<1.39$, no multiple loops, at most 3 continuous unpaired bases and at most 7 unpaired bases in the mature miRNAs. All precursor secondary structures were recalculated with p-values of 0.01 and the '-p' option at $37^{\circ} \mathrm{C}$. The frequency distribution of MFE and the empirical distribution were modeled using a modified sigmoid function

$$
\begin{aligned}
& x=M F E / \text { length } \\
& f(x)=\frac{a}{b+e^{x * c}}
\end{aligned}
$$

Where length represented the length of the precursor, $a=1.339 \mathrm{e}-12, b=2.7783 \mathrm{e}-13$, and $c=45.843$ were the fitting parameters. The stability was calculated using $f$ $(x)$. The selection criterion was $f(x)>-4.42$. Finally, 8,494 pseudo pre-miRNAs were collected as the negative dataset.

While training the model miPlantMat, we collected pre-miRNAs that were not intercepted by mature miRNAs or miRNAs* from the primary pre-miRNAs. We treated these pre-miRNAs as pseudo samples for miPlantMat. The sequences either contained real samples for miPlantMat or were contained in real samples for miPlantMat. If a base was paired with another base on the opposite strand of the stem in the pseudo premiRNAs, the paired base was collected in the pre-miRNAs to maintain the stem-loop structure. Consequently, the pseudo samples must be similar to the real samples for the classification to be significant.

\section{Features of plant miRNAs and pre-miRNAs}

Recent studies have demonstrated that the primary sequence and secondary structure of plant pre-miRNAs exhibit many features that can be used to classify real/ pseudo plant pre-miRNAs. Because the sequences of almost all mature miRNAs are located in the stems of the corresponding pre-miRNAs, the sequences either begin from miRNAs and end in miRNAs or form a stem-loop structure. Based on these features, mature miRNAs can be located in pre-miRNAs. The stemloop of Solanum lycopersicum miR-166b was used as an example. The stem-loop without interception was treated as a real sample in the MiPlantPre model of miPlantPreMat. The stem-loop with interception is treated as a real sample in the miPlantMat model of miPlantPreMat.

Structural characteristics are also very important for identifying real/pseudo pre-miRNAs. 32 structured triplet composition features are defined in triplet-SVM (including the frequencies of " $G\left(\left({ }^{\prime \prime}\right.\right.$ and " $C(($. ", which are extracted from the pre-miRNAs. A left bracket "(" indicates that a paired nucleotide is located near the 5 '-end and can be paired with another nucleotide at the 3'-end, and the corresponding nucleotide at the 3 '-end is indicated using a right bracket ")". As in previous studies, "(" and ")" were treated equally. A dot"." indicates that a nucleotide does not pair with a nucleotide on opposing end. These 32 features were extracted from stems and are denoted as "G(((_S" and "C((._S", etc.

29 global and intrinsic folding features were extracted from secondary structures of real/pseudo pre-miRNAs defined in miPred. These features include the following: (i) $\% \mathrm{G}+\mathrm{C}$ content and 16 dinucleotide frequencies defined as $\% \mathrm{XY}$, where $\mathrm{X}, \mathrm{Y}$ in $\{\mathrm{A}, \mathrm{C}, \mathrm{G}, \mathrm{U}\}$; (ii) adjusted base pairing propensity denoted as dP [20]; (iii) the MFE of folding denoted as dG [21]; (iv) the adjusted base pair distance denoted as $\mathrm{dD}$ [22]; (v) the adjusted Shannon entropy denoted as dQ [23]; (vi) the MFE index denoted as MFEI1 and MFEI2 [24], a topological descriptor of the degree of compactness denoted as $\mathrm{dF}$; and (vii) 5 normalized variants of $\mathrm{dP}, \mathrm{dG}, \mathrm{dQ}, \mathrm{dD}$ and $\mathrm{dF}$ denoted as $z \mathrm{P}, \mathrm{zG}, \mathrm{zQ}, \mathrm{zD}$ and $\mathrm{zF}$, respectively [25].

19 features defined in microPred [26] include the following: (i) seven base pair-related features that are denoted as $|A-U| / L,|G-C| / L,|G-U| / L$, the average number of base pairs per stem (Avg_BP_Stem), $\%(\mathrm{~A}-\mathrm{U}) / \mathrm{n} \_$stems, $\%(\mathrm{G}-\mathrm{C}) / \mathrm{n} \_$stems and $\%(\mathrm{G}-\mathrm{U}) /$ n_stems; (ii) the MFE index denoted as MFEI3 and MFEI4; (iii) four RNA fold-related features, such as the normalized ensemble free energy (NEFE); the frequency of the MFE structure denoted as Freq; structural thermodynamic features such as the structural entropy dS and $\mathrm{dS} / \mathrm{L}$; the structural enthalpy $\mathrm{dH}$ and $\mathrm{dH} / \mathrm{L}$; and the melting energy of the structure, denoted as $\mathrm{Tm}$ and $\mathrm{Tm} / \mathrm{L}$, where $\mathrm{L}$ represents the length of the pre-miRNA sequences and $n \_$stems represents the number of stems in the secondary structure.

3 features defined in PlantMiRNAPred [27] include: (i) the MFE index denoted as MFEI5 and MFEI6; (ii) the average number of mismatches per 21-nt window, which is calculated as Avg_mis_num = tot_mismatches/n_21nts, where tot_ mismatches is the total number of mismatches in the 21-nt sliding window and $n \_21 n t s$ is the number of sliding windows in a stem.

69 novel features proposed in our study include the following: (i) MFE Index 7: MFEI7 $=\mathrm{MFE} / \% \mathrm{G}+\mathrm{C}_{-}$ Begin_n_21nts, where $\% G+C_{-}$Begin_ $n \_21 n t s$ is the GC content in the first 21 bases of the stems; MFE Index 8: MFEI8 $=$ MFE $/ \% G+C$ C_End_n_21nts, where $\% \mathrm{G}+\mathrm{C} \_$End_n_21nts is the GC content in the last 21 bases of the stems; MFE Index 9: MFEI9= MFE/avg_ mis_num_n_21nts, where avg_mis_num_n_21nts is the average number of mismatches per 21-nt window; (ii) Mis_num_begin: the nucleotide is not paired with a nucleotide on the opposing terminus in the first 21 bases of the stems; (iii) Mis_num_end: the nucleotide 
is not paired with a nucleotide on the opposing terminus in the last 21 bases of the stems. Because the miRNAs and miRNAs* are stable, it is necessary to determine the position of the mature miRNAs in the corresponding pre-miRNAs; and (iv) to obtain improved classification, features that reflect both the sequence and secondary structure of the real/pseudo pre-miRNAs and that aid in determining the position of the mature miRNA in the pre-miRNA were needed. In addition to the features extracted above, 64 new features including the frequencies of "G((_begin" and "A.(._end" were extracted from the beginning and end of pre-miRNAs. Because almost all mature miRNAs were located in stems, these 64 features were extracted from stems and were denoted as "G((_begin_S" and "A.(._end_S", etc.

152 features belonging to six groups were selected, as shown in Table 1. MFEI1, MFEI2, MFEI3, MFEI4, MFEI5, MFEI6, MFEI7, MFEI8, and MFEI9 were considered MFE-related features. 20 features that reflect the proportion of adjacent bases and the $\mathrm{G}$ and $\mathrm{C}$ content of bases were used as sequence-related features. 6 thermodynamic features were used as mfold-related features. Seven types of base pairing were used as base pair-related features. 96 features were triple-related. 14 features calculated by RNAfold were used as RNAfoldrelated features. Secondary structures and thermodynamic parameters were obtained using the ViennaRNA package. All RNAfold-related features were extracted using the RNAfold program using the '-p' option at $37^{\circ} \mathrm{C}$. For consistency, every parameter was scaled in the range from -1 to 1 .

\section{SVM and miPlantPreMat classifier}

We chose SVM as our classification paradigm in this research based on its excellent generalization ability. For a given dataset $X_{n}, x_{i} \in X_{n}(i=1,2, \ldots, N)$, each element in the dataset has a corresponding label $\gamma_{i}(-1$ or +1 , representing the two classes to be classified; +1 represents real samples whereas -1 represents pseudo samples). A decision function is given by the SVM classifier

$$
f(x)=\operatorname{sgn}\left(\sum_{i=1}^{N} \gamma_{i} \alpha_{i} K\left(x, x_{i}\right)+b\right)
$$

Where $\gamma_{i}$ is the class label of the $i$-th element, $\alpha_{i}$ is the coefficient to be learned, $K$ is the kernel function, and $b$ is the offset. $\alpha_{i}$ is obtained by maximizing

$$
\sum_{i=1}^{N} \alpha_{i}-\frac{1}{2} \sum_{i, j=1}^{N} \alpha_{i} \alpha_{j} \gamma_{i} \gamma_{j} K\left(x_{i}, x_{j}\right)
$$

If the value of $f(x)$ is greater than zero, the label assigned to data $x$ is +1 ; otherwise, the assigned label is -1 .

The LIBSVM package (version 3.1) [28] was used in our study. To obtain the best performance, the penalty parameter $C$ and the RBF kernel parameter $\gamma$ were calculated using grid search strategy.

MiPlantPreMat was proposed based on SVM, as illustrated in Figure 2. A total of 3,126 non-redundant plant pre-miRNAs with single stem-loops were collected from miRBase release 19.0 and used as the positive dataset. A total of 8,494 non-redundant sequence segments with stem-loop structures similar to real pre-miRNAs that were not previously reported as pre-miRNAs were collected and used as the negative dataset. (i) A total of 2,000 positive and 2,000 negative samples were randomly collected for use in training the miPlantPre model of MiPlantPreMat; (ii) 152 features were extracted from the primary sequences and secondary structures of pre-miRNA stems; (iii) redundant features were eliminated, and the informative feature subset was selected using B-SVM-RFE; (iv) miPlantPre was trained with the selected 47 features; (v) 3,835 sequence segments from the 3,126 pre-miRNAs mentioned above were collected and used as the positive dataset. The sequence segments extended from the beginning of the mature miRNAs to the end of the miRNAs*, from both the 5 ' and 3 ' arms. A total

\begin{tabular}{|c|c|c|}
\hline Classification & Number & Features \\
\hline MFE-related & 9 & MFEI1 ${ }^{2}, M F E I 2^{2}, M F E I 3^{3}, M F E I 4^{3}, M F E I 5^{4}, M F E I 6^{4}, M F E I 7^{5}, M F E I 8^{5}, M F E I 9^{5}$ \\
\hline Sequence-related & 20 & $\% A A, \% A C$, etc. $^{2}(16), \% G+C^{2}$, Avg_mis_num ${ }^{4}$ Mis_num_begin ${ }^{5}$, Mis_num_end ${ }^{5}$ \\
\hline Mfold-related & 6 & $d S^{3}, d S / L^{3}, d H^{3}, d H / L^{3}, T m^{3}, T m / L^{3}$ \\
\hline Base-pair -related & 7 & $|A-U| / L^{3},|C-G| / L^{3},|G-U| / L^{3}$, Avg_BP_Stem ${ }^{3}, \%(A-U) / n \_s t e m s^{3}, \%(G-C) / n \_s t e m s^{3}$ \\
\hline Triple-related & 96 & 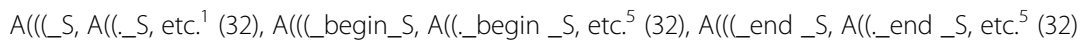 \\
\hline RNAfold-related & 14 & $\mathrm{dP}^{2}, \mathrm{dG}^{2}, \mathrm{dD}^{2}, \mathrm{dQ}^{2}, \mathrm{dF}^{2}, \mathrm{zP}^{2}, \mathrm{zG}^{2}, \mathrm{zD}^{2}, \mathrm{zQ}^{2}, \mathrm{zF}^{2}, \mathrm{NEFE}^{3}$, Freq $^{3}$, Diversity $^{3}$, Diff $^{3}$ \\
\hline
\end{tabular}

Table 1 Selected pre-miRNA features

${ }^{1}$ Features extracted in triplet-SVM.

${ }^{2}$ Features extracted in miPred.

${ }^{3}$ Features extracted in microPred.

${ }^{4}$ Features extracted in plantMiRNAPred.

${ }^{5}$ Features extracted in miPlantPreMat. 


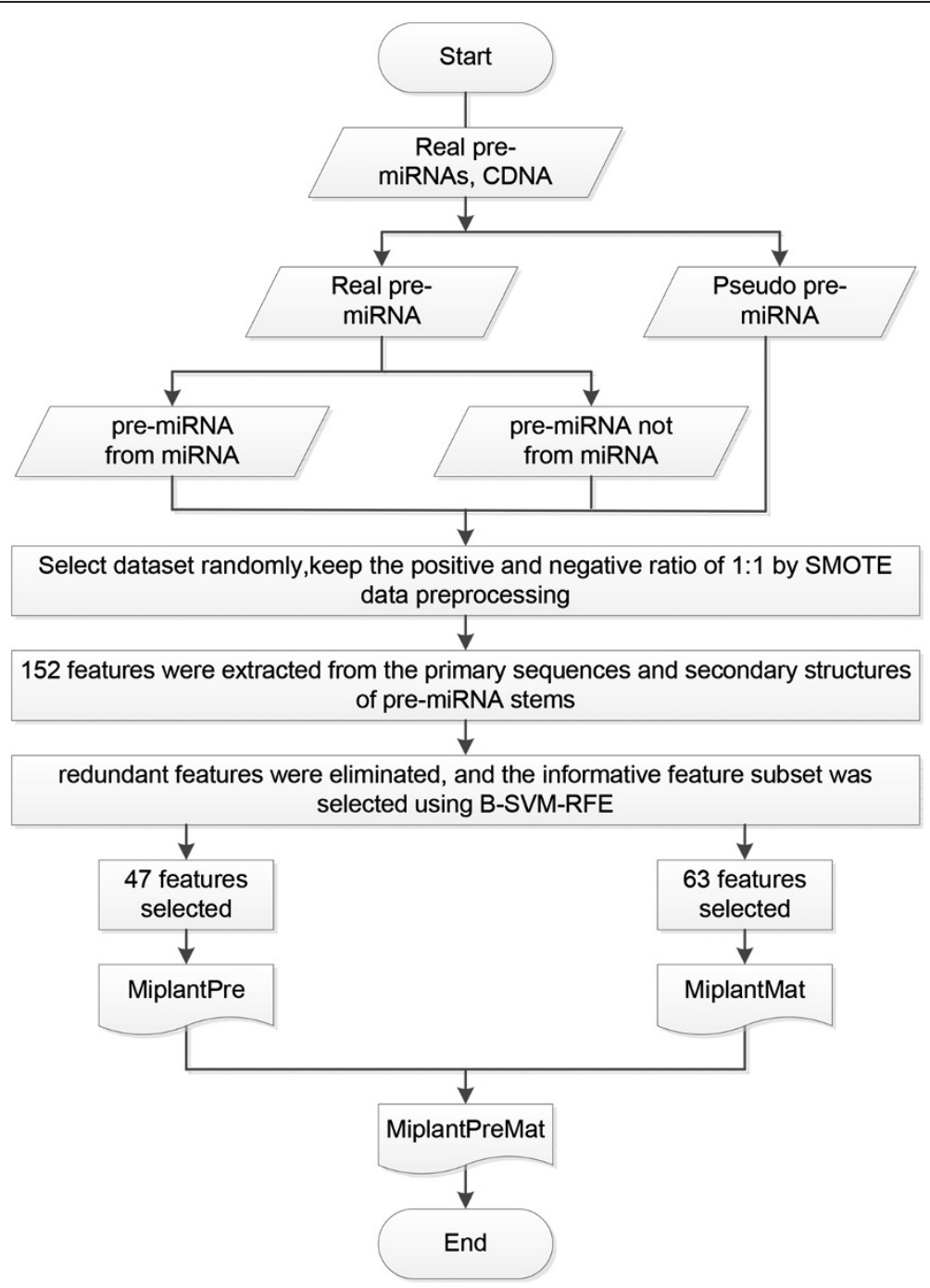

Figure 2 Flow chart of the classification model miPlantPreMat for use with plant miRNAs. Construction of SVM classifier MiPlantPreMat based on feature selection and sample selection was shown.

of 39,428 sequence segments from the same pre-miRNAs, which were not previously included in the positive dataset, were longer than $55 \mathrm{nt}$, and comparable stem-loop structures were collected for use as the negative dataset. Randomly, 1,000 positive and 5,000 negative samples were collected and preprocessed using SMOTE to train the miPlantMat model of MiPlantPreMat, keep the positive and negative ratio of $1: 1$; (vi) miPlantMat was trained using the selected 63 features using the same method; and (vii) an integrated MiPlantPreMat model was constructed by combining MiPlantPre and MiPlantMat. The detailed feature extraction and selection of the SVM model are shown in Additional file 1.

\section{Feature subset selection}

Feature subset selection is used to choose a group of informative features that retain the most information from the original data, screen out redundant features and distinguish each sample in the dataset. A total of 152 features were selected without considering redundancy and correlation with class. SVM-RFE [29,30] was used for subset selection in our study.

SVM-RFE is a simple and efficient feature selection algorithm that ranks features according to the SVM classification results. The evaluation function is biased toward subsets that contain features that are highly correlated with class. Irrelevant features should be ignored because they will be poorly correlated with class. Feature subset selection can be summarized as follows: (i) input training examples $X_{0}=\left[x_{1}, x_{2}, \ldots, x_{n}\right]^{\mathrm{T}}$ together with their class labels $y=\left[y_{1}, y_{2}, \ldots, y_{n}\right]^{\mathrm{T}}$; (ii) initialize the subset of surviving features $s=[1,2, \ldots, 152]$ and the features ranked list $r=$ [], repeat until $s=$ []; (iii) restrict the training examples to those exhibiting good feature indices $X=X_{0}(:, s)$ 
and train the classifier $\alpha=S V M$ - $\operatorname{train}(X, y)$; (iv) compute the weight vector of dimension length $(s), w=\sum_{k} \alpha_{k} y_{k} x_{k}$; (v) compute the ranking criteria $c_{i}=\left(w_{i}\right)^{2}$ for all $i$; (vi) find the feature with the smallest ranking criterion $f=\operatorname{argmin}$ (c) and update the feature ranked list $r=[s(f), r]$; (vii) eliminate the feature with the smallest ranking criterion $s=s$ (1: $f-1, f+1$ : length $(s)$ ); and (viii) find the classifier $\alpha$ and the subset of trained classifiers $\alpha$.

A total of 152 features without redundancy were extracted under the initial conditions. These features represent a sample but do not fully consider the relationship between the attributes during extraction and classification. SVM-RFE can dynamically calculate attribute weights, sort each attribute, and fulfill feature selection. However, once the attributes are sorted to the bottom, they can no longer participate in subsequent attribute weight calculations. Because the training number is different each time, the properties calculated under different SVM classification space attribute weights also differ. Therefore, sorting of the calculated weights of less important properties may be overshadowed by properties with a higher weight. Here, we propose the use of a B-SVM-RFE method that is based on the attribute of Information Gain [31] (IG).

Information entropy is an important concept underlying information gain. For a classification system, the possible values of a category are $C_{1}, C_{2}, \ldots, C_{n}$, where $P\left(C_{1}\right), P\left(C_{2}\right)$, ..., $P\left(C_{n}\right)$ represent the probabilities of each category and $n$ represents the total number of categories. The information entropy of the classification system is expressed as:

$$
H(C)=-\sum_{i=1}^{n} P\left(C_{i}\right) * \log _{2} P\left(C_{i}\right)
$$

Information gain is reliant on characteristic $t$. When calculating the differences in information entropy between when characteristic $t$ exists and when it does not, the increased amount of information obtained is the information gain.

Characteristics of $t$ included in the system of information entropy can be obtained. When $t$ does not belong to the system, feature $t$ is treated as a constant. Then, the problem can be seen as computing the conditional entropy with constant $t$

$$
H(C \mid T)=P(t) H(C \mid t)+P(\bar{t}) H(C \mid \bar{t})
$$

Where $T$ is the characteristic, $t$ indicates the presence of characteristic $T$, and $\bar{t}$ indicates the absence of characteristic $T$. Then, the information gain of characteristic $T$ can be calculated as follows:

$$
I G(T)=H(C)-H(C \mid T)=\sum_{C, T} p(C T) \log _{2} \frac{p(C T)}{p(C) p(T)}
$$

A total of 2,000 real samples and 2,000 pseudo samples were chosen from the data pool using progressive sampling. The information gain and SVM-RFE ranking of the 4,000 samples regarding the 152 features are listed in Table 2.

First, the information gain of each attribute and the SVM-RFE ranking were calculated. Then, the existing set $s=[1,2, \ldots, 152]$ and ranking set $r=[]$ of the properties were updated. The SVM model was trained, and the property ranking was sent to ranking set $r$. If properties existed that yielded higher information gain than the property with the lowest weight in $s$, then the property with the highest information gain in $r$ would be sent to the existing set $s$. Then, the SVM model was trained again. If the cross validation error at this time was better than that obtained during the previous run, then the property with the highest information gain in $r$ would be sent back to $s$. The existing set and the ranking set would be updated and used to train the SVM model again. If the cross validation error at this time was not better than that obtained during the previous run, then the property would be sent back to $r$. The SVM model was trained until no property was present in existing set $s$. Finally, the property set with the best cross validation error was selected for use. The process is illustrated in Figure 3.

During feature selection, the 5-fold cross validation recognition rate (LooErrorRate) and independent test error recognition rate (TestErrorRate) were used to determine the best feature set. When B-SVM-RFE was used to train the model with 5 -fold cross validation, the parameter of the penalty coefficient $C$ and the kernel function parameter $g$ were set to the default values. When tested using an independent test set, the grid search method was used to determine the best parameters. The process used to determine the best feature set is shown in Figure 4.

In this paper, 2,000 real samples and 2,000 pseudo samples were used to train the SVM model; 1,000 real samples and 1,000 pseudo samples were used in the test set, and the principle of the fence was used to verify that no sample appeared repeatedly both in the training and testing sets. The feature set $F_{1}, F_{2}, \ldots, F_{152}$ represents the number of corresponding properties of the sample space selected using B-SVM-RFE. The best classification rate was obtained using this feature subset. The classification rate was tested using 5 -fold cross validation, and the LooErrorRate and TestErrorRate for SVM-RFE and BSVM-RFE obtained in this experiment are provided in Table 3 . The lowest 5 -fold cross validation recognition rate (LooErrorRate) and the independent test error recognition rate (TestErrorRate) were $2.42 \%$ and $7.04 \%$, respectively. In this paper, this subset of 47 features was selected to train miPlantPreMat. 
Table 2 Information gain of each attribute and SVM-RFE ranking

\begin{tabular}{|c|c|c|c|c|c|}
\hline Feature & IG & SVM-RFE rank & Feature & IG & SVM-RFE rank \\
\hline$d P$ & 0.78628 & 1 & U..._S & 0.09652 & 58 \\
\hline MFEI5 & 0.77982 & 2 & C..._s_end & 0.0933 & 103 \\
\hline$z P$ & 0.75613 & 3 & $G(((S S$ & 0.07866 & 30 \\
\hline MFEI7 & 0.68656 & 54 & A..._S_end & 0.07662 & 74 \\
\hline MFE/8 & 0.66704 & 48 & $C(((S)$ & 0.072 & 13 \\
\hline$\ldots$ & $\cdots$ & & & $\ldots$ & $\ldots$ \\
\hline$\% G G$ & 0.12375 & 38 & $G(((S S$ & 0.07866 & 30 \\
\hline MFEl6 & 0.1227 & 25 & A..._S_end & 0.07662 & 74 \\
\hline$d H / L$ & 0.11855 & 77 & $C(((S)$ & 0.072 & 13 \\
\hline$\% C U$ & 0.11651 & 8 & $\%(G-C) / n \_s t e m s$ & 0.07079 & 44 \\
\hline MFE/4 & 0.11603 & 15 & G..._S_begin & 0.06746 & 93 \\
\hline G..._S_end & 0.11563 & 34 & $\% G C$ & 0.06041 & 28 \\
\hline C....S & 0.11034 & 139 & U.(._S & 0.05969 & 101 \\
\hline$d F$ & 0.10372 & 127 & A..._S_begin & 0.05779 & 53 \\
\hline
\end{tabular}

Normal plant pre-miRNAs are 60-150 nt in length. Only one miRNA is located in the pre-miRNA; however, more than one pseudo miRNA can be obtained from the pre-miRNA. If the same positive and negative data rates were to be applied to miPlantMat and miPlantPre, the obtained information might reflect pseudo samples from miPlanMat that would be of little use to the classification model and possibly increase the false positive rate. In this paper, the positive and negative data rates were set at 1:5, indicating that if one positive data sample were selected, then five negative data samples would also be selected. This data rate is closer to that of the original data samples and reflects the distribution features of the data samples. However, this result illustrates the class imbalance problem. The data classification is biased toward the negative class, potentially resulting in a high false negative rate. To solve these problems, the SMOTE [32] method was used for data processing.

Two potential methods can be used to solve the classification problem for unbalanced data. The first method is to balance the dataset, and the second is to improve the performance of the machine learning algorithm on specific issues. In 2002, Chawla proposed a method that improved the fitting sample problem caused by traditional classification. The main idea behind SMOTE was to increase rare class samples by joining the closer rare class samples to the "simulation" samples and then increasing the number of rare class samples to approach a dense sample number. The specific experimental steps are as follows:

i. determine the sampling ratio $N$ and the number of rare class samples $x$; identify $k$ nearest neighbors for each rare class sample; identify $N$ points from the original $x$ class samples and their $x^{*} k$ similar samples; ii. identify each $k$ nearest neighbors from the selected $N$ rare class samples and the original rare class samples, and then identify $k$ neighbor samples of the $(x+N)^{*} k$ class samples from the original sample; new rare class samples randomly generate $N$ points as the rare generated class sample;

iii. new rare class samples are added to the original training set, thus forming a new training data set,

$$
x_{\text {new }}=x+\operatorname{rand} *(y[i]-x)
$$

Where $i=1,2, \ldots, N$; rand is a random number between 0 and $1 ; x_{\text {new }}$ represents the new sample; $x$ represents the original sample; and $y[i]$ represents the $i$-th neighbor of $x$.

\section{Evaluation method}

The classification model was trained using the informative feature subset and the training samples using 5-fold crossvalidation and default values for $g$ and $C$. Datasets were optimized for $g$ and $C$ using the grid selection approach recommended by LIBSVM. The accuracy of the prediction result was evaluated based on the number of true positives $(T P)$, false positives $(F P)$, true negatives $(T N)$ and false negatives $(F N)$. The sensitivity $(S E)$, specificity $(S P)$, geometric mean $(G m)$ and total prediction accuracy $(A c c)$ used to assess the prediction system were calculated according to the following definitions:

$$
S E=\frac{T P}{T P+F N}
$$




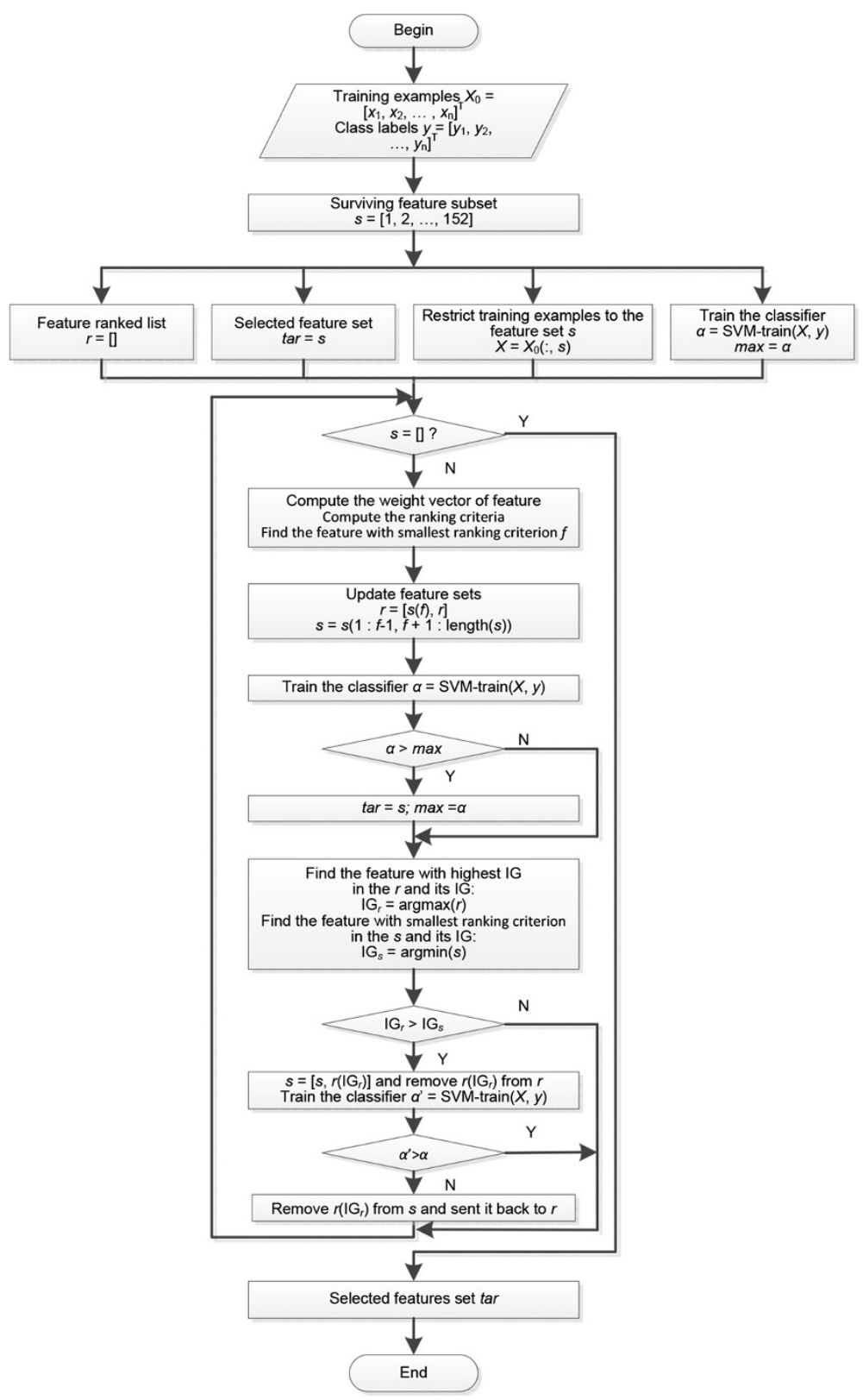

Figure 3 Flow chart of B-SVM-RFE feature selection. Feature subset was selected using B-SVM-RFE. This method was combined by SVM-RFE and information gain. The final feature subset for miPlantPreMat was obtained.

$$
\begin{aligned}
& S P=\frac{T N}{F P+T N} \\
& A c c=\frac{T N+T P}{T P+F P+F N+T N} \\
& G m=\sqrt{S E \times S P}
\end{aligned}
$$

Where $S E$ is the proportion of positive samples (real pre-miRNAs) that are correctly classified as pre-miRNAs, and $S P$ is the proportion of negative samples (pseudo premiRNAs) that are correctly classified as pre-miRNAs.

\section{Results}

\section{The results of feature subset selection}

To obtain the highest classification performance, three subset selection methods were used in this paper: Principal Components Analysis (PCA), Correlation-based Feature Subset Selection (CFS) [33] and B-SVM-RFE. Additionally, three machine-learning methods were used in this paper: naiveBayes (NBC) [34], RandomForest (RF) [35] and SVM. Finally, the subset collected using B-SVM-RFE and trained using SVM was chosen because it performed better than the other selection 


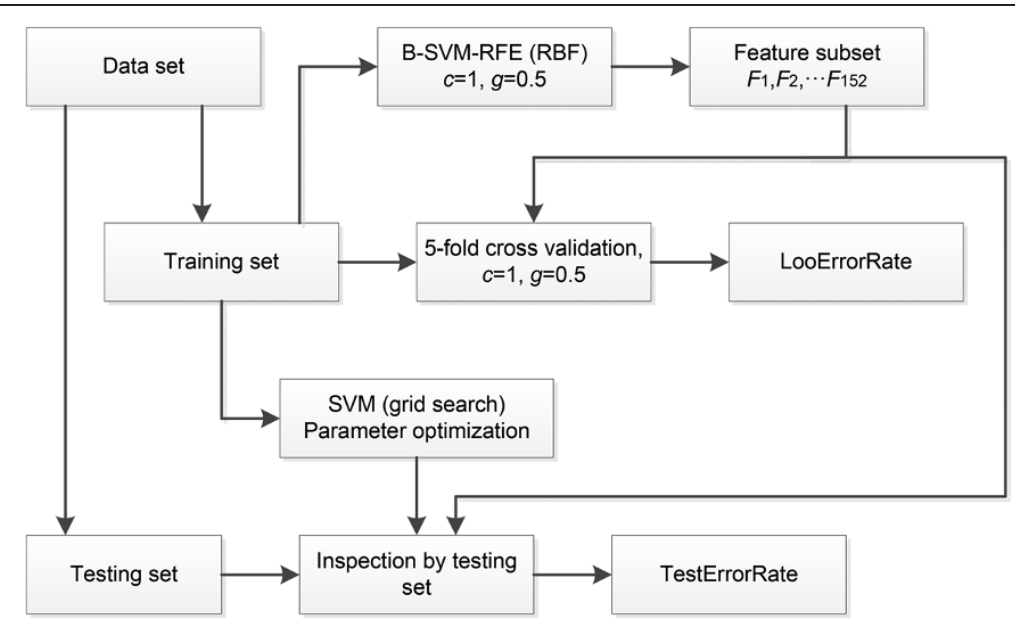

Figure 4 Determination of the best feature subset. Two indicators named LooErrorRate and TestErrorRate were used for the best subset evaluation. The LooErrorRate was calculated with 5 -fold cross validation model. The TestErrorRate was calculated by independent training set and testing set with optimized parameters. The parameters of penalty coefficient $c$ and the kernel function parameter $g$ were obtained by grid search method.

methods. Subsets containing 47 features used for miPlantPre and 63 features used for miPlantMat were acquired. The selected features were ranked as shown in Additional files 2 and 3. Among the selected features, dS, dH and Tm are related to mfold. A number of studies have verified that the stem-loop structures of plant pre-miRNAs are thermodynamically stable [36]. A(((_S, G..._S and C..._S are triple-related. Studies have shown that local adjacent structures can be applied to distinguish real pre-miRNAs from pseudo miRNAs [37]. The features $\% A A, \% U C$ and $\% G+C$ are related to sequence. Because pre-miRNAs are composed of nucleotide sequences that have unique characteristics, the sequence composition of pre-miRNAs is useful for classification [12].
Table 4 shows that the SVM using subset selection method B-SVM-RFE has the best performance.

\section{Parameter subset selection compared with other methods} To test the efficiency of our model, we compared miPlantPre with five existing models (Triplet-SVM, MiPred, miPred, miRabela and microPred). Table 5 shows that miPlantPre exhibited better performance than the existing models in terms of sensitivity $(S E)$, specificity $(S P)$, geometric mean $(G m)$ and total prediction accuracy (Acc) while using fewer features.

Tests on different plant species

Pre-miRNAs of Arabidopsis thaliana (ath), Glycine max (gma), Oryza sativa (osa), Physcomitrella patens (ppt),

Table 3 LooErrorRate and TestErrorRate of SVM-RFE and B-SVM-RFE

\begin{tabular}{|c|c|c|c|c|}
\hline \multirow[t]{2}{*}{ Feature number } & \multicolumn{2}{|l|}{ SVM-RFE } & \multicolumn{2}{|l|}{ B-SVM-RFE } \\
\hline & LooErrorRate & TestErrorRate & LooErrorRate & TestErrorRate \\
\hline 1 & 21.13 & 26.53 & 21.13 & 26.53 \\
\hline 2 & 11.40 & 21.01 & 11.40 & 21.01 \\
\hline 3 & 9.91 & 20.94 & 9.91 & 20.94 \\
\hline$\cdots$ & $\cdots$ & $\cdots$ & $\cdots$ & $\cdots$ \\
\hline 46 & 3.04 & 7.15 & 2.72 & 7.15 \\
\hline 47 & 2.84 & 7.34 & 2.42 & 7.04 \\
\hline 48 & 2.72 & 7.14 & 2.72 & 7.14 \\
\hline$\cdots$ & $\cdots$ & $\cdots$ & $\cdots$ & $\cdots$ \\
\hline 150 & 3.00 & 8.17 & 3.00 & 8.17 \\
\hline 151 & 3.19 & 8.29 & 3.19 & 8.29 \\
\hline 152 & 3.30 & 7.30 & 3.30 & 7.30 \\
\hline
\end{tabular}


Table 4 Classification results based on different feature subsets using three methods

\begin{tabular}{|c|c|c|c|c|c|c|c|}
\hline \multirow[t]{2}{*}{ Model } & \multirow[t]{2}{*}{ ML method } & \multirow{2}{*}{$\begin{array}{l}\text { Feature subset } \\
\text { selection method }\end{array}$} & \multirow{2}{*}{$\begin{array}{l}\text { Feature } \\
\text { number }\end{array}$} & \multicolumn{4}{|c|}{ Classification results (\%) } \\
\hline & & & & $S E$ & $S P$ & Acc & Gm \\
\hline \multirow[t]{12}{*}{ miPlantPre } & \multirow[t]{4}{*}{ NBC } & PCA & 76 & 92.2 & 92.6 & 92.4 & 92.4 \\
\hline & & CFS & 20 & 93.9 & 97.8 & 95.8 & 95.8 \\
\hline & & B-SVM-RFE & 47 & 93.8 & 98.6 & 96.2 & 96.2 \\
\hline & & All features & 152 & 92.9 & 98.0 & 95.4 & 95.4 \\
\hline & \multirow[t]{4}{*}{ RF } & PCA & 76 & 93.5 & 95.3 & 94.4 & 94.4 \\
\hline & & CFS & 20 & 95.0 & 97.6 & 96.3 & 96.3 \\
\hline & & B-SVM-RFE & 47 & 95.3 & 97.7 & 96.5 & 96.5 \\
\hline & & All features & 152 & 95.3 & 97.7 & 96.5 & 96.5 \\
\hline & \multirow[t]{4}{*}{ SVM } & PCA & 76 & 94.9 & 99.2 & 97.0 & 97.0 \\
\hline & & CFS & 20 & 94.3 & 99.1 & 96.7 & 96.7 \\
\hline & & B-SVM-RFE & 47 & 95.5 & 99.1 & 97.2 & 97.2 \\
\hline & & All features & 152 & 93.9 & 98.5 & 96.2 & 96.2 \\
\hline \multirow[t]{12}{*}{ miPlantMat } & \multirow[t]{4}{*}{ NBC } & PCA & 71 & 88.6 & 82.3 & 85.5 & 85.4 \\
\hline & & CFS & 40 & 93.2 & 74.8 & 83.6 & 83.5 \\
\hline & & B-SVM-RFE & 63 & 89.8 & 88.4 & 89.1 & 89.1 \\
\hline & & All features & 152 & 91.7 & 79.3 & 85.5 & 85.3 \\
\hline & \multirow[t]{4}{*}{ RF } & PCA & 71 & 93.2 & 73.2 & 83.2 & 82.6 \\
\hline & & CFS & 40 & 89.2 & 89.1 & 89.2 & 89.2 \\
\hline & & B-SVM-RFE & 63 & 89.7 & 88.6 & 89.2 & 89.2 \\
\hline & & All features & 152 & 86.6 & 84.4 & 85.5 & 85.5 \\
\hline & \multirow[t]{4}{*}{ SVM } & PCA & 71 & 88.6 & 84.3 & 86.4 & 86.4 \\
\hline & & CFS & 40 & 90.6 & 87.5 & 89.1 & 89.1 \\
\hline & & B-SVM-RFE & 63 & 92.9 & 88.7 & 90.8 & 90.8 \\
\hline & & All features & 152 & 87.1 & 81.6 & 84.4 & 84.4 \\
\hline
\end{tabular}

Medicago truncatula (mtr), Sorghum bicolor (sbi), Arabidopsis lyrata (aly), Zea mays (zma) and Solanum lycopersicum (sly) were used to compare the efficiency of miPlantPre with three widely used methods. To show that the false positive rate was sufficiently low, a negative dataset was used to test the efficiency of miPlantPre (Table 6).

miPlantPre performed better than Triplet-SVM and microPred for most species. The sub-sequences in premiRNAs that begin from the miRNAs and end at the miRNAs or that form a stem-loop structure were selected. These nine species were also used to test the efficiency of miPlantMat regarding miRNA classification. The results are shown in Table 7. These pre-miRNAs and miRNAs were published in miRBase release 20.0.

The accuracies found using these species were all greater than $87 \%$, demonstrating the utility of miPlantMat for classification in plants. Moreover, the false positive rates (FPRs) obtained were all lower than $13.36 \%$

\section{Searching miRNAs in Solanum lycopersicum}

Studies have shown that miRNAs are relatively conserved during the evolutionary process. Therefore, some miRNAs exhibit conservative evolutionary relationships among species [38]. There are two basic principles behind our method. One is that homologous fragments can be identified according to sequence or structural similarity. The second is that new miRNAs can be discovered using known miRNAs [39]. To date, 77 Solanum lycopersicum mature miRNAs have been reported in miRBase (Release 21, 2014.6.26). Through studying these 77 miRNAs, which are distributed among 31 miRNA families.

MiRNAs of the same family may be found in a large number of species. In this study, known plant miRNAs were used to identify potential miRNAs in Solanum lycopersicum. First, genome-scale fragments might contain similarities to known miRNAs. If less than 3 mismatches were found for two related sequences, we considered the sequences similar. The KMP [40] algorithm was used to 
Table 5 Comparison of miPlantPre against other methods

\begin{tabular}{|c|c|c|c|c|c|c|c|c|c|}
\hline \multirow[t]{2}{*}{ Methods } & \multicolumn{2}{|c|}{ Training dataset } & \multicolumn{2}{|c|}{ Testing dataset } & \multirow{2}{*}{$\begin{array}{l}\text { Features } \\
\text { selected }\end{array}$} & \multicolumn{4}{|c|}{ Classification results (\%) } \\
\hline & pos & neg & pos & neg & & $S E$ & $S P$ & Acc & Gm \\
\hline Triplet-SVM & 163 & 168 & 30 & 1000 & 32 & 93.30 & 88.10 & 90.66 & 90.66 \\
\hline MiPred & 163 & 168 & 263 & 265 & 34 & 89.35 & 93.21 & 91.26 & 91.26 \\
\hline miPred & 200 & 400 & 123 & 146 & 34 & 84.55 & 97.97 & 91.01 & 91.01 \\
\hline miRabela & \multicolumn{4}{|c|}{ Not given clearly in the article } & & 71.00 & 97.00 & 82.99 & 82.99 \\
\hline microPred & \multicolumn{4}{|c|}{ SMOTE + outer-5-fold-cV } & 21 & 90.02 & 97.28 & 93.58 & 93.58 \\
\hline plantMiRNAPred & \multicolumn{4}{|c|}{ outer-5-fold-cv } & 68 & 91.93 & 97.84 & 94.84 & 94.84 \\
\hline miPlantPre & \multicolumn{4}{|c|}{ outer-5-fold-cv } & 47 & 95.50 & 98.82 & 97.16 & 97.16 \\
\hline
\end{tabular}

compare known miRNAs on a genome-wide scale. A series of potential miRNAs was obtained for comparison. Structural information regarding putative miRNAs was obtained using RNAfold. Potential pre-miRNAs were obtained by identifying stem-loop-containing fragments. Several potential miRNAs with hairpins were obtained by limiting the minimum number of base pairings in the hairpin structure to $19, \% \mathrm{G}+\mathrm{C}>0.242$ and $<0.825$, MFEI $>0.522$ and $<1.39$, not allowing multiple loops, limiting continuous unpaired bases to 3 , allowing no more 7 unpaired bases on a mature miRNA and not allowing any uncertain bases ("N") in the pre-miRNA. Finally, 522 miRNA were identified as real miRNAs by testing their pre-miRNAs using our classification model.

In this study, we denoted the length of the sequence as $l$, the number of sequences as $n$, the length of the miRNA sequence as $k$, and the number of the miRNA sequences as $m$. Then, the average time complexity is $O$ $(n * m * l * k)$.

This time complexity was unacceptable. In this study, an algorithm was developed based on to the SEED algorithm [41] and the KMP algorithm. The known plant
miRNA sequence was divided into four nearly equal sequence lengths. We compared these four sequences with the complete genome sequence of Solanum lycopersicum to identify similar fragments. When a matching pattern occurred, we completed the miRNA pairing with the corresponding positioning of the sequence. Sequences with less than 3 mismatches were saved. The average time complexity is $O\left(4 *(k / 4+l) * k^{*} n^{*} m / 4^{k / 4}\right)$.

Using the method described above, several potential miRNAs were obtained. Structural information regarding the miRNAs was obtained using RNAfold. Potential pre-miRNAs were obtained by identifying stem-loopcontaining fragments, and potential pre-miRNAs with hairpins were obtained using the following criteria: stability above $-4.42, \% \mathrm{G}+\mathrm{C}$ content between $30 \%$ and $70 \%$, less than 6 bases of mature miRNA that are not complementary with the other arm, no gaps in the complementary miRNA strand, no uncertain base ("N") in the pre-miRNA, and less than 3 consecutive non-complementary bases.

As an important economic crop, Solanum lycopersicum is not only nutritious but also has various physiological

Table 6 The classification accuracy of four methods for the pre-miRNA of several plants species and for the negative dataset

\begin{tabular}{|c|c|c|c|c|}
\hline \multirow{2}{*}{$\begin{array}{l}\text { Plant species \& } \\
\text { negative dataset }\end{array}$} & \multicolumn{4}{|l|}{ Methods } \\
\hline & Triplet-SVM & MiPred & plantMiRNAPred & miPlantPreMat \\
\hline aly & 94.29 & 96.19 & 96.19 & 99.05 \\
\hline ath & 91.75 & 90.72 & 92.78 & 96.91 \\
\hline gma & 91.18 & 92.65 & 93.93 & 95.89 \\
\hline mtr & 85.90 & 88.46 & 89.74 & 90.60 \\
\hline osa & 92.31 & 95.10 & 95.10 & 95.10 \\
\hline ppt & 88.44 & 91.16 & 97.96 & 98.64 \\
\hline sbi & 93.38 & 97.79 & 96.99 & 98.53 \\
\hline sly & 97.14 & 100.00 & 100.00 & 100.00 \\
\hline zma & 89.74 & 97.44 & 97.44 & 98.29 \\
\hline neg & 94.80 & 97.80 & 98.20 & 98.60 \\
\hline
\end{tabular}


Table 7 The classification results obtained using miPlantMat for various pre-miRNA datasets

\begin{tabular}{lll}
\hline Plant species & \multicolumn{2}{l}{ Classification results (\%) } \\
\cline { 2 - 3 } & Accuracy & FPR \\
\hline aly & 89.46 & 9.46 \\
ath & 87.84 & 10.53 \\
gma & 89.50 & 13.36 \\
mtr & 87.67 & 12.22 \\
osa & 88.96 & 10.31 \\
ppt & 90.98 & 10.46 \\
sbi & 89.02 & 9.53 \\
sly & 89.87 & 8.36 \\
zma & 91.42 & 10.93 \\
\hline
\end{tabular}

functions that are relevant to the exploitation and development of plant resources. Currently, only 77 Solanum lycopersicum miRNAs are reported in miRbase, far less than the actual number of Solanum lycopersicum miRNAs. In this study, we found 522 Solanum lycopersicum miRNAs in the complete genome. Their sequence and ID in other plants are shown in Additional file 4.

Figure 5 shows the number of predicted members which is more than 4 and the corresponding reported number in Solanum lycopersicum. (i) some of the predicted miRNAs have been reported as Solanum lycopersicum miRNAs in miRBase release 21.0; (ii) the more the members of miRMA family predicted in other plants, the more the members verified of this family in Solanum lycopersicum, it is concluded that their trends are similar with respect to the number of miRNA family; (iii) the number of miRNAs verified in Solanum lycopersicum is still less than the predicted number. Therefore, new miRNAs remain to be verified in the future.

\section{Discussion}

In this study, a new classifier, miPlantPreMat, was developed for predicting plant pre-miRNAs and miRNAs. MiPlantPreMat was developed by analyzing existing miRNA prediction methods, combining the characteristics of plant pre-miRNAs, extracting features, selecting features and training samples to achieve efficient and effective classification. Importantly, 152 features were extracted to distinguish the hairpins of real/pseudo pre-miRNAs based on the characteristics of plant premiRNAs and miRNAs. After selecting the best subset for classification, 47 informative features were selected for use with miPlantPre, and 63 informative features were selected for use with miPlantMat. The accuracy, sensitivity and specificity of miPlantPreMat were all greater than $95 \%$ in terms of pre-miRNA classification and greater than $85 \%$ in terms of miRNA classification. Additionally, 522 potential miRNAs with stem-loop structures were found in the Solanum lycopersicum genome. The results of our study might prove useful for subsequent biological experiments.

\section{Conclusions}

A comparison method was developed based on miRNA homology. Some miRNAs with low or specific expression patterns might not be found using this method. In the future, we intend to develop better classification models that can identify miRNAs with low and specific expression levels.

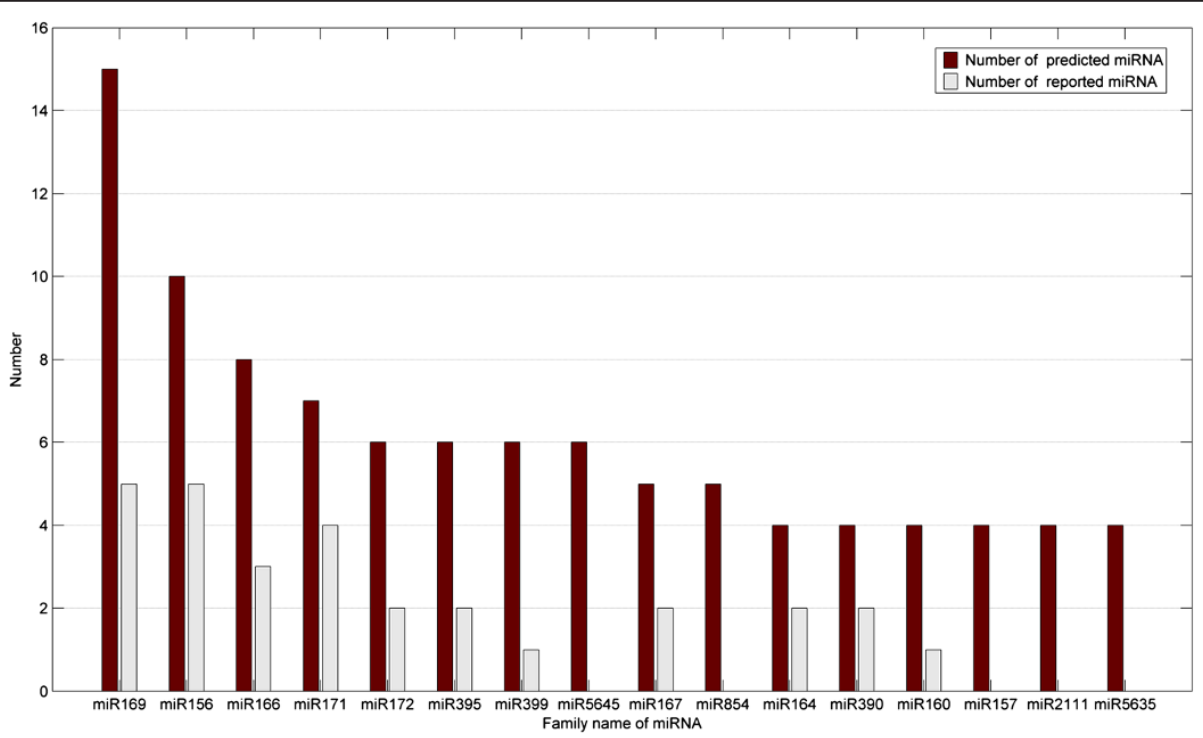

Figure 5 Number of predicted members and reported number in Solanum lycopersicumis. The number of predicted members which is more than 4 and the corresponding reported number in Solanum lycopersicum. 


\section{Additional files}

Additional file 1: Feature extraction and selection of the SVM model.

Additional file 2: Table S1. Ranking of the selected 47 features used in miPlantPre.

Additional file 3: Table S2. Ranking of the selected 63 features used in miPlantMat.

Additional file 4: Table S3. Sequence and ID in other plants of 522 predicted miRNAs.

\section{Competing interests}

The authors declare that they have no competing interests.

\section{Authors' contributions}

JM and DL developed the methods and CS wrote the codes and implemented most of the experiments under the guide of JM. YL provided helpful insights into method development and guided the forming of the ideas. JM, DL and CS wrote the manuscript. All the authors read and approved the manuscript.

\section{Acknowledgements}

The current study was supported by the National Natural Science Foundation of China (Nos. 31272167, 61472061, and 31471880) and the Teaching Reform Foundation for Graduate School of Dalian University of Technology (No.JGXM201223).

\section{Author details}

${ }^{1}$ School of Computer Science and Technology, Dalian University of Technology, Dalian, Liaoning 116023, China. ${ }^{2}$ School of Life Science and Biotechnology, Dalian University of Technology, Dalian, Liaoning 116023, China.

Received: 30 July 2014 Accepted: 11 December 2014

Published online: 30 December 2014

\section{References}

1. He L, Hannon GJ: MicroRNAs: small RNAs with a big role in gene regulation. Nat Rev Genet 2004, 7(5):522-531.

2. Julia SR, Jacek K, Edyta K, Piotr K: Structural basis of microRNA length variety. Nucleic Acids Reaearch 2010, 39(1):257-268.

3. Chatterjee $\mathrm{S}$, Grobhans $\mathrm{H}$ : Active turnover modulates mature microRNA activity in caenorhabditis elegans. Nature 2009, 461:546-549.

4. Baek D, Villen J, Shin C, Camargo FD, Gygi SP, Bartel DP: The impact of microRNAs on protein output. Nature 2008, 7209(455):64-71.

5. Brennecke J, Hipfner DR, Stark A, Russell RB, Cohen SM: Bantam encodes a developmentally regulated microRNA that controls cell proliferation and regulates the proapoptotic gene hid in Drosophila. Cell 2003, 113(1):25-36.

6. Xu PZ, Vernooy SY, Guo M, Hay BA: The Drosophila microRNA Mir-14suppresses cell death and is required for normal fat metabolism. Curr Biol 2003, 13(9):790-795.

7. Shusei S, Michael E, Robert B, Li CB: The tomato genome sequence provides insights into fleshy fruit evolution. Nature 2012, 485:635-641.

8. Mendes ND, Freitas AT, Sagot MF: Current tools for the identification of miRNA genes and their targets. Nucleic Acids Res 2009, 37(8):2419-2433.

9. Lim LP, Lau NC, Weinstein EG: The microRNAs of Caenorhabditis elegans. Genes Dev 2003, 17(8):991-1008.

10. Lai EC, Tomancak P, Williams RW: Computational identification of Drosophila microRNA genes. Genome Biol 2003, 7(4):R42.

11. Huang TH, Fan B, Rothschild MF: MiRFinder: an improved approach and software implementation for genome-wide fast microRNA precursor scans. BMC Bioinformatics 2007, 8:341

12. Bartel DP: MicroRNAs: genomics, biogenesis, mechanism, and function. Cell 2004, 116(2):281-297.

13. Xue CH, Li F, He T: Classification of real and pseudo microRNA precursors using local structure-sequence features and support vector machine. BMC Bioinformatics 2005, 6:310-316.

14. Yousef M, Nebozhyn M, Shatkay H: Combining multi-species genomic data for microRNA identification using a Naïve Bayes classifier. Bioinformatics 2006, 22(11):1325-1334.
15. Jiang $P, W u H$, Wang $W$ : MiPred: classification of real and pseudo microRNA precursors using random forest prediction model with combined features. Nucleic Acids Res 2007, 35(Web Server issue)):W339-344.

16. Gordon L, Chervonenkis AY, Gammerman AJ, Shahmuradov IA, Solovyev W: Sequence alignment kernel for recognition ofpromoter regions. Bioinformatics 2003, 19(15):1964-1971.

17. Lim LP, Lau NC, Weinstein EG: The microRNAs of Caenorhabditis elegans. Genes Dev 2003, 17(8):991-1008.

18. Meng J, Shi L, Luan YS: Plant microRNA-target interaction identification model based on the integration of prediction tools and support vector machine. Plos One 2014, 9(7):e103181.

19. Lai EC, Tomancak P, Williams RW: Computational identification of Drosophila microRNA genes. Genome Biol 2003, 7(4):R42

20. Jones-Rhoades MW, Bartel DP: Computational identification of plant microRNAs and their targets including a stress-induced miRNA. Mol Cell 2004, 14(6):787-799.

21. Schultes EA, Hraber PT, LaBean TH: Estimating the contributions of selection and self-organization in RNA secondary structure. J Mol Evol 1999, 49(1):76-83.

22. Wojciechowski P, Formanowicz P, Blazewicz J: Reference Alignment Based Methods for Quality Evaluation of Multiple Sequence Alignment-A Survey. Current Bioinformatics 2014, 9(1):44-56.

23. Maji S, Garg D: Hybrid Approach Using SVM and MM2 in Splice Site Junction Identification. Current Bioinformatics 2014, 9(1):76-85.

24. Moorthy $K$, Saberi M, Deris S: A review on missing value imputation algorithms for microarray gene expression data. Current Bioinformatics 2014, 9(1):18-22.

25. Zhang BH, Pan XP, Cox SB: Evidence that miRNAs are different from other RNAs. Cell Mol Life Sci 2006, 63(2):246-254.

26. Batuwita R, Palade V: microPred: effective classification of pre-miRNAs for human miRNA gene prediction. Bioinformatics 2009, 25(8):989-995.

27. Xuan P, Guo M, Liu X: PlantMiRNAPred: efficient classification of real and pseudo plant pre-miRNAs. Bioinformatics 2011, 27(10):1368-1376.

28. Chih-Chung C, Chih-Jen L: LIBSVM: A Library for Support Vector Machines. ACM Trans Intell Syst Technol 2011, 2(3):27 (27 pp.).

29. Guyon I, Weston J, Barnhill S: Gene selection for cancer classification using support vector machines. Machine learning 2002, 46(1-3):389-422.

30. Guyon I, Elisseeff A: An introduction to variable and feature selection. The J Mach Learn Res 2003, 3:1157-1182.

31. Kent JT: Information gain and a general measure of correlation. Biometrika 1983, 70(1):163-173.

32. Chawla NV, Bowyer KW, Hall LO: SMOTE: synthetic minority over-sampling technique. arXiv preprint 2011, 11(6):1813-1819.

33. Hall MA: Correlation-based feature selection for machine learning. Hamilton: The University of Waikato; 1999

34. John GH, Langley P: Estimating continuous distributions in Bayesian classifiers. In: Proceedings of the Eleventh conference on Uncertainty in artificial intelligence. San Francisco: Morgan Kaufmann Publishers Inc., 1995: 338-345.

35. Breiman L: Random Forests. Machine Learning 2001, 45:5-32.

36. Xuan P, Guo M, Liu X, Huang Y, Li W, Huang Y: PlantMiRNAPred: efficient classification of real and pseudo plant pre-miRNAs. Bioinformatics 2011, 27(10):1368-1376.

37. Bonnet E, Wuyts J, Rouzé P, Van de Peer Y: Evidence that microRNA precursors, unlike other non-coding RNAs, have lower folding free energies than random sequences. Bioinformatics 2004, 20(17):2911-2917.

38. Umesono K, Evans RM: Determinants of target gene specificity for steroid/thyroid hormone receptors. Cell 1989, 57(7):1139-1146.

39. Lund E, Sheets MD, Imboden SB: Limiting Ago protein restricts RNAi and microRNA biogenesis during early development in Xenopus laevis. Genes Dev 2011, 25(11):1121-1131.

40. Regnier M: Knuth-Morris-Pratt algorithm: an analysis. In: Mathematical Foundations of Computer Science 1989. New York: Springer, 1989: 431-444.

41. Ohler U, Yekta S, Lim LP: Patterns of flanking sequence conservation and a characteristic upstream motif for microRNA gene identification. RNA 2004, 10(9):1309-1322. 